\title{
Fatores associados a não-utilização de anticoncepcionais na adolescência ${ }^{\star}$
}

\section{Factors associated with the non-utilization of contraceptives in adolescence}

\author{
Evely Boruchovitch** \\ BORUCHOVITCH, E. Fatores associados a não utilização de anticoncepcionais na adolescência. \\ Rev. Saúde públ., S.Paulo, 26: 437-43,1992. Elaborou-se revisão crítica da literatura sobre os fatores \\ associados ao não uso de anticoncepcionais, na adolescência. Procurou-se integrar resultados de \\ pesquisa, tanto no sentido de uma maior compreensão da vulnerabilidade do adolescente para \\ problemas como a gravidez indesejada e a AIDS, quanto para o desenvolvimento de uma educação \\ sexual mais efetiva e realista para esta faixa etária.
}

Descritores: Adolescência. Comportamento contraconceptivo. Educação sexual.

\section{Introduçáo}

A adolescência é vista pela literatura como um período caracterizado por intensa necessidade de explorar e experimentar o contexto em que se vive. Evidências indicam que essa necessidade de exploração e de experiência tornam o adolescente mais vulnerável ao engajamento em comportamentos que envolvem riscos pessoais (Irwin e Millstein ${ }^{24}$, 1986).

Se por um lado, experimentar e explorar são tendências naturais do desenvolvimento do adolescente (Baumrind ${ }^{4}, 1985$ ), por outro torna-se cada vez mais evidente que os adolescentes de hoje engajam-se mais e mais em comportamentos que possam ter consequiências negativas, a curto e a longo prazo, para uma vida adulta sadia.

E dentro deste contexto de risco que o comportamento sexual do adolescente vem sendo pensado e entendido. A maioria dos casos de gravidez, na adolescência, são indesejados, e cada vez mais, também, aumentam os casos de AIDS, nessa faixa etária. Tendo-se em vista a importância do uso de anticoncepcionais para a prevenção e redução de problemas sérios que afetam a vida sexual do adolescente, a proposta do presente trabalho é de rever a literatura, criticamente, a respeito dos fatores que estão associados a uma postura de risco e de descuido que, geralmente, $o$ adolescente tem

\footnotetext{
* Estudo elaborado com o apoio financeiro da Coordenação de Aperfeiçoamento de Pessoal de Nivel Superior CAPES (Ref. $\left.n^{2} 10099 / 88-7\right)$.

* Doutoranda do Departamento de Psicologia Educacional da "University of Southem California - Los Angeles, USA".
}

Separatas/Reprints: E. Boruchovitch-3419 Keystone ave \# 5, Los Angeles, CA 90034 USA. para com a sua própria sexualidade, bem como integrar resultados de pesquisa em termos de implicaçōes para uma educação sexual mais realista e voltada para as necessidades do adolescente.

A literatura relativa aos fatores associados a não-utilização de anticoncepcionais, na adolescência, é caracterizada por diferentes perspectivas. A maioria das pesquisas nessa área concentra-se predominantemente na relação do não-uso de anticoncepcionais na adolescência e casos de gravidez indesejada, visto ser a AIDS um problema mais recente.

Alguns estudos investigam o conhecimento do adolescente sobre fisiologia reprodutiva e gravidez, bem como suas atitudes e crenças relativas ao uso de anticoncepcionais (Dembo e Lundell ${ }^{13}$, 1979; Darabi e col.11, 1982; Black e Deblassie ${ }^{5}$, 1985; Morrison ${ }^{30}, 1985$ ). Outros estudos, ainda nessa linha de pesquisa, concentram-se no levantamento do conhecimento e atitudes de préadolescentes e adolescentes em relação a AIDS (DiClemente e col. ${ }^{14}$, 1986; Rebello e col. ${ }^{36}, 1989$; Monteiro e col. ${ }^{29}, 1991$ ).

Em outras investigaçð̃es, predomina-se a preocupação com a influência de diferenças psicológicas individuais, variáveis motivacionais e de personalidade no uso de anticoncepcionais, por parte do adolescente (Miller ${ }^{27}$, 1976; Rosen e Ager $^{38}$, 1981; Morrison ${ }^{30}$, 1985; Pestrak e Martin ${ }^{32}$, 1985; Alvarez e col. ${ }^{1}$, 1987; Hart e Hilton ${ }^{22}$, 1988; Romig e Bakken ${ }^{37}$, 1990).

Outras pesquisas têm sido conduzidas no sentido de investigar a força das variáveis biológicas (ex. momento da entrada na puberdade) na utilização de anticoncepcionais, na adolescência (Simkins $^{39}$, 1984; Pestrak e Martin 32, 1985; Irwin e Millstein ${ }^{24}$, 1986; Steinberg ${ }^{40}$, 1989; Phinney e col. $^{33}, 1990$ ). 
Existem ainda, investigações que se preocupam com a influência que o novo tipo de funcionamento cognitivo, caracteristico da adolescência, possa ter na busca e prática de anticoncepcionais, nesta faixa etária (Elkind ${ }^{16}, 1967$; Piaget ${ }^{34}, 1972$; Namerow e col. ${ }^{31}$, 1987; Burger e Burns ${ }^{7}$, 1989).

Outros estudos dedicam-se, também, ao entendimento das relações do adolescente com a familia e com os parceiros, e a implicação desses relacionamentos no uso de anticoncepcionais, nessa faixa etária (Davis ${ }^{12}$, 1980; Simkins ${ }^{39}$, 1984; PolitO'Hara e Kahn ${ }^{35}$, 1985; Fisher ${ }^{17}$, 1986; Baumrind $^{4}$, 1987; Romig e Bakken ${ }^{37}$, 1990).

Como a utilização de anticoncepcionais na adolescência faz parte de um comportamento complexo e multifacetário, estudos em diferentes direçð̄es oferecem contribuições que se enriquecem e se complementam. Nesse sentido, será apresentada uma síntese dos principais resultados e das diferentes linhas de pesquisa, acima mencionadas.

Conhecimento do adolescente sobre fisiologia reprodutiva, gravidez e AIDS: atitudes e crenças relativas ao uso de anticoncepcionais

De acordo com Dembo e Lundell ${ }^{13}$ (1979), um dos fatores associados ao não-uso de anticoncepcionais na adolescência, é a falta de conhecimento do adolescente acerca de questőes sexuais. Estudos mais recentes indicam que este grupo continua mal informado, apresentando falta de compreensão de assuntos como o ciclo menstrual, o tempo de fertilidade e o processo de concepção (Darabi e col. ${ }^{11}$, 1982). Os adolescentes possuem crenças errôneas de que a fertilidade não começa com a primeira menstruação, o que acarreta incapacidade de perceber que o risco de gravidez existe desde a primeira relação sexual e tendem a achar que é necessário que eles sejam mais velhos para que a gravidez ocorra (Morrison $^{30}, 1985$; Black e Deblassie ${ }^{5}$, 1985).

No que diz respeito a AIDS, a maioria dos adolescentes possui conhecimento de que a atividade sexual e o uso comum de seringas se constituem nos principais veículos para contrair a doença. No entanto, de modo geral os adolescentes têm pouca consciência de que o uso de preservativos reduz o risco de transmissão dessa doença (DiClemente e col. ${ }^{14}$, 1986). Confundem formas de contrair a AIDS, acreditando que aperto de mãos e proximidade física a alguém doente leva ao contágio, sentindo-se ainda capazes de diferenciar portadores da doença daqueles que não o são, e apresentam dificuldades para entender que alguém aparentemente assintomático possa ser portador de uma doença fatal (DiClemente e col. ${ }^{14}, 1986$; Rebello e col. ${ }^{36}$, 1989; Monteiro e col. ${ }^{29}$, 1991). Como men- ciona Strunin e Hingson ${ }^{42}$ (1987), apesar de terem havido mudanças no comportamento casual do adolescente devido ao medo da AIDS, ocorreram poucas mudanças em relação ao comportamento sexual, o que realmente pode transmitir a doença.

Quanto ao uso de anticoncepcionais, estudos apontam que adolescentes são mal informados sobre os métodos anticoncepcionais existentes, porém, a maioria é capaz de identificar pelo menos um desses métodos. Adolescentes do sexo feminino, geralmente sabem mais sobre anticoncepcionais do que os do sexo masculino. Os adolescentes tendem, também, a apresentar atitudes negativas sobre o uso de anticoncepcionais que é visto como um fator de interferência no prazer sexual, transformando o ato sexual em algo não natural e préplanejado. Possuem, ainda, crenças errôneas de que a maioria dos métodos anticoncepcionais é incompatível com a baixa frequiência e naturalidade de suas relaçōes sexuais, bem como idéias de que o uso de anticoncepcionais é de responsabilidade de seu parceiro (Morrison ${ }^{30}, 1985$ ).

Diferenças individuais e o uso de anticoncepcionais: variáveis motivacionais e de personalidade

Estudos sugerem que determinadas características de personalidade contribuem para o uso ou não de anticoncepcionais, na adolescência. Os adolescentes que optam por medidas anticoncepcionais, geralmente apresentam nivel maior de maturidade de ego. Os que possuem comportamento sexual mais desprotegido possuem dificuldades em desenvolver relaçōes íntimas com adultos e colegas, inabilidade de planejar o futuro, maior impulsividade e baixo nível de tolerância à frustração (Hart e Hilton 22, 1988; Romig e Bakken ${ }^{37}, 1990$; Pestrak e Martin ${ }^{32}, 1985$ ).

Tem-se demonstrado, também, que variáveis como ansiedade, "locus" de controle, auto-estima e nível de aspiração influenciam o uso de anticoncepcionais na adolescência. Estudos apontam para uma possivel relação curvilínea entre ansiedade e uso de anticoncepcionais, nessa faixa etária. $\mathrm{A}$ ansiedade moderada atuaria então como facilitadora para utilização de anticoncepcionais, (Miller ${ }^{27}$, 1976; Morrison ${ }^{30}$, 1985). Em relação ao "locus" de controle, indivíduos que costumam considerar que a causa do que lhes acontece é externa e fora do seu próprio controle tendem a utilizar menos anticoncepcionais (Rosen e Ager ${ }^{38}, 1981$ ). Da mesma forma, estudos sugerem que quanto maior a autoestima da pessoa, maior também é a sua tendência a procurar práticas sexuais mais seguras. No que se refere ao nível de aspiração educacional, quanto mais alto o nível de aspiração educacional entre os adolescentes, maior é o uso de anticoncepcionais 
por parte dos mesmos. De acordo com Alvarez e col. ${ }^{1}$ (1987), adolescentes grávidas tendem a ser mais imediatistas, mais devotadas ao presente, e mostram menos preocupação ou perspectiva diante do futuro.

\section{Variáveis biológicas: 0 momento em que a pu- berdade começa}

A idade média em que os adolescentes vêm entrando no processo de puberdade tem diminuído consideravelmente, atingindo seu limite inferior (Steinberg ${ }^{40}, 1989$ ). O entrar na puberdade, mais cedo, geralmente acarreta um amadurecimento biológico que não necessariamente coincide com o amadurecimento cognitivo e emocional, o que se constitui, portanto, fator de risco para uma iniciação sexual prematura e suas negativas conseqüências (Simkins ${ }^{39}, 1984$ ). Adolescentes do sexo feminino que entram na puberdade mais cedo do que a média, apresentam tendência maior a ter experiências sexuais precoces (Pestrak e Martin ${ }^{32}$ 1985; Phinney e col. $\left..^{33}, 1990\right)$. Os do sexo masculino que atingem a puberdade tanto antes como depois da média possuem também mais chances de engajamento em comportamentos desviantes e de risco à própria vida. Os que se engajam em um tipo de comportamento potencialmente autodestrutivo têm, concomitantemente, maior probabilidade de estarem simultaneamente vinculados a outros comportamentos de risco (Irwin e Millstein ${ }^{24}$, 1986; Brooks-Gunn e col. ${ }^{6}, 1988$ ).

\section{Variáveis cognitivas}

O funcionamento cognitivo da adolescência é caracterizado, segundo Piaget ${ }^{34}$ (1972), pelo desenvolvimento do pensamento operacional formal. Esse modo de funcionamento cognitivo capacita o adolescente a lidar com proposiçōes abstratas, pensar hipoteticamente e sobre possibilidades futuras. De acordo com Elkind ${ }^{16}$ (1967), - acesso ao estágio operacional formal leva ao surgimento de um fenômeno chamado egocentrismo. Este fenômeno faz do adolescente alguém excessivamente preocupado com si mesmo, leva-o a se considerar único na história e a achar que ninguém viveu o que ele está vivendo no momento. Conseqüentemente, o adolescente passa a ter dificuldades de perceber que é tão vulnerável a quaisquer eventos e perigos quanto qualquer outra pessoa. Essa percepção de invulnerabilidade do adolescente consigo próprio exerce um certo impacto no seu engajamento em comportamentos que envolvem riscos pessoais. Mais especificamente, no que se concerne ao uso de anticoncepcionais, Burger e Burns? (1988) en- contraram entre adolescentes universitárias a relação de que quanto maior a percepção de invulnerabilidade, menor a procura e uso efetivo de anticoncepcionais. Namerow e col. ${ }^{31}$ (1987) chamam atenção para o fato de que os adolescentes são guiados para uso de anticoncepcionais, do ponto de vista cognitivo, muito mais em função de uma estimativa subjetiva da probabilidade de que algo negativo the aconteça, do que pela consciência realista de risco.

\section{Relaçōes familiares e comunicação com parceiros}

Baumrind ${ }^{4}$ (1987) estudou diferentes estilos de pais e suas respectivas influências no envolvimento do adolescente, em comportamentos potencialmente autodestrutivos. Pais que são mais afetivos, democráticos e que promovem o diálogo entre eles e os filhos são mais capazes de proteger seus filhos contra um engajamento em comportamentos perigosos. Romig e Bakken ${ }^{37}$ (1990) mencionam que famílias rígidas tendem a aumentar o risco de ocorrência de gravidez indesejada na adolescência.

Do ponto de vista social, mudanças na estrutura da família e o concomitante aumento das taxas de divórcio parecem também contribuir para a iniciação sexual precoce do adolescente, que passa mais tempo na rua e é menos supervisionado por seus familiares (Steinberg ${ }^{40}, 1989$ ).

Apesar do adolescente se sentir capaz de ter relações sexuais com alguém, ele não necessariamente se sente à vontade para discutir com o seu parceiro questoes ligadas ao uso de anticoncepcionais.

De acordo com Byme ${ }^{8}$ (1983), o uso de anticoncepcionais na adolescência envolve cinco etapas: primeiramente 0 adolescente precisa ter a informação científica a respeito de anticoncepcionais, em seguida, os adolescentes precisam reconhecer a probabilidade de seu engajamento em alguma relação sexual. Essa etapa é árdua, pois eles tendem a ter dificuldades de planejar atividades sexuais com antecedência, bem como também a exibirem atitudes negativas sobre relações sexuais programadas. Como terceiro passo, Byme $^{8}$ (1983) menciona que os adolescentes precisam selecionar, obter e saber usar corretamente o método escolhido. A quarta fase implica que 0 adolescente comunique a sua decisão e escolha ao seu parceiro. A quinta e última etapa implica o uso efetivo e competente do anticoncepcional. Byme ${ }^{8}$ (1983) salienta que as barreiras contra o uso de anticoncepcional podem ocorrer em qualquer um desses momentos.

Polit-O'Hara e Kahn ${ }^{35}$ (1985) mencionam como fortes indicadores para o uso de anticoncepcionais, 
a comunicação efetiva e a competência verbal do adolescente. Quanto maior é esta competência, maiores são as chances de que o adolescente consiga convencer seu parceiro da importância do uso de anticoncepcionais.

\section{Implicações para uma educação sexual mais realista}

Compreender os fatores associados a nãoutilização de anticoncepcionais na adolescência é essencial, dada a importância que o uso de anticoncepcionais tem para a prevenção dos sérios problemas que afetam a vida sexual do adolescente.

A maioria dos estudos nessa área são de natureza correlacional. Apesar de serem extremamente úteis para a identificação de fatores associados ao não-uso de anticoncepcionais na adolescência, carecem de poder explanatório para especificar o porque da não-utilização de anticoncepcionais, nessa faixa etária. Embora as relações causais específicas ainda não tenham sido estabelecidas e a maioria dos estudos ter tido como foco principal o uso de anticoncepcionais para a prevenção da gravidez indesejada, pode-se perceber que existe um corpo sólido de conhecimento, nessa área, o que pode ser instrumentalizado a favor de uma educação sexual mais efetiva na adolescência.

É evidente que os educadores e profissionais da área de saúde não podem olhar para a adolescência como se ela não fosse uma etapa do desenvolvimento onde há risco de vida para o adolescente. Não importa se correr risco é necessariamente normativo ou importante para o desenvolvimento do adolescente, se apenas adolescentes se arriscam mais do que adultos, ou ainda se adolescentes definem risco de modo diferente dos adultos, como aventam Furby e Beyth-Maron ${ }^{19}$ (1990). Cabe, como sugerem Irwin e Millstein ${ }^{24}$ (1986), que se pense em maneiras para ajudar o adolescente a refletir sobre o seu próprio processo de desenvolvimento, sensibilizando-o para os riscos reais que ele corre, sem contudo aniquilá-lo, no seu processo de busca de novas experiências, extremamente necessário, para formação de sua identidade e autonomia.

Com as mudanças na estrutura social da familia, a escola passa a ser um forte contexto para o desenvolvimento de uma educação sexual, que promova no adolescente um senso de auto-responsabilidade e compromisso para a sua própria sexualidade.

Educadores sexuais devem ficar atentos para adolescentes do sexo feminino que atingem a puberdade precocemente. Essas adolescentes devem ser um foco de atenção especial dos programas de educação sexual, já que a maturação biológica pre- coce as torna mais vulneráveis ao engajamento prematuro, em atividades sexuais. Como sugerem Ingersoll e Orr ${ }^{23}$ (1989), estratégias preventivas devem concentrar esforços em diferentes comportamentos de risco simultaneamente, dado que estes se correlacionam, na adolescência. A iniciação sexual precoce é geralmente associada com o uso de álcool e drogas e com risco de evasão escolar (Brooks-Gunn e col. ${ }^{6}, 1988$ ).

No que toca às diferenças individuais, dado que na adolescência comportamentos potencialmente autodestrutivos se exacerbam, e devido à freqüente associação de mais de um tipo de comportamento de auto-risco, nessa faixa etária, faz-se necessário que sejam conduzidos estudos longitudinais mais profundos a respeito das diferenças individuais que possam estar associadas ao engajamento em comportamentos potencialmente perigosos em idades anteriores. É possível que tais diferenças sejam muito mais determinantes de uma predisposição para o engajamento em comportamentos de auto-risco, do que anteriormente se havia pensado. Assim sendo, a descoberta e detecção desses fatores, precocemente, podem abrir importantes "avenidas" para a prevenção do desenvolvimento do comportamento de autodestruição, como uma postura de vida.

No que se refere a aumentar o nivel de informação científica do adolescente sobre questōes sexuais, as evidências sugerem que os cursos de educação sexual não têm se mostrado eficazes em ajudar adolescentes a transformarem a informação científica em comportamentos saudáveis. Isto se deve ao fato da educação sexual ter se concentrado maciçamente na transmissão de informação científica, o que de uma maneira geral é possível para o adolescente adquiri-la por intermédio de outras fontes. De fato a relação entre aquisição de conhecimento e adoção de medidas anticoncepcionais, na adolescência é bem pequena (Chilman ${ }^{10}$, 1983). Isto não equivale a dizer que a informação científica não é importante, porém, como aponta Monsour e Stewart ${ }^{28}$ (1973), ela sozinha não é capaz de alcançar o senso de autoconsciência, nem tampouco levar o adolescente a uma compreensão emocional de sua própria sexualidade.

Marlach e Kerr ${ }^{26}$ (1983) estudaram as principais necessidades dos adolescentes em relação à educação sexual e observaram que este grupo revelou preferência por uma educação sexual que envolva discussões de grupo, com pessoas do mesmo sexo, realizadas por um profissional de fora da escola, que enfoque de modo aberto e que se sinta confortável em relação a questōes sexuais. Esses adolescentes enfatizaram ainda o desejo de uma educação sexual mais voltada para os aspectos humanos e positivos da sexualidade ao invés de 
única e exclusivamente ser centrada na prevenção de conseqüências negativas associadas a ela. Neste sentido, as evidências indicam que mensagens voltadas para o desenvolvimento de um conceito mais realista a respeito do risco (por oposição a mensagens que promovam o medo), bem como informações precisas de como o comportamento perigoso pode ser mudado mostram-se bem mais efetivas, na redução do comportamento de risco e no compromisso com a mudança (Brooks-Gunn e col. ${ }^{6}, 1988$; Catania e col. ${ }^{9}, 1990$ ).

Além de alteraçð̃es no contexto e no conteúdo dos cursos de educação sexual para adolescentes, os educadores sexuais precisam levar em conta, para preparação e elaboração dos cursos, não apenas como o adolescente pensa sobre questões sexuais, suas concepçð̄es errôneas e atitudes relativas ao uso de anticoncepcionais, mas também as peculiaridades de seu funcionamento cognitivo. Maiores esforços devem ser feitos no sentido de compreender que o adolescente, geralmente, percebe riscos e sente-se vulnerável, muito mais em função de probabilidades subjetivas do que reais. Perceber-se realisticamente susceptível a riscos, constitui-se fator de extrema importância para tomada de decisões em direção a prevenção e redução dos mesmos (Goch$\operatorname{man}^{20,21}$, 1971, 1986; Catania e col. ${ }^{9}, 1990$ ). Atenção a esta faceta do funcionamento cognitivo do adolescente é de extrema relevância para o sucesso da educação sexual, pois como ressaltam Pestrak e Martin ${ }^{32}$ (1985), se por um lado não se tem a certeza de que todos adolescentes alcançam o pensamento formal, por outro é evidente que tamanha sofisticação cognitiva não é garantia de um comportamento sexual responsável por parte do adolescente.

É também importante que os educadores sexuais atentem para a variável "locus" de controle e compreendam as atribuições causais do adolescente a respeito dos problemas que afetam a sua sexualidade. $O$ adolescente precisa acreditar que a prevenção de problemas como a AIDS e a gravidez indesejada está dentro de seu próprio controle.

Cursos de educação sexual devem ensinar e preparar adolescentes também para discutir sobre anticoncepcionais antes da primeira relação sexual. De acordo com Polit-O'Hara e Kahn ${ }^{29}$ (1985), cursos de educação sexual devem ter como proposta melhorar a capacidade de comunicação interpessoal do adolescente, pois esta terá um impacto direto no uso de anticoncepcionais, nessa faixa etária. Muito importante é ainda que os cursos de educação sexual promovam também o desenvolvimento da capacidade de tomada de decisões do adolescente, visto ser a capacidade de decidir um componente chave para adoção, por parte do adolescente, de uma postura de auto-responsabilidade pela sua própria sexualidade.
Tem sido sugerido, também, que programas de educação sexual na adolescência utilizem técnicas onde o adolescente possa aumentar seu senso de auto-eficácia (confiança na sua própria capacidade de reduzir riscos e mudar), praticar diversos papéis, exercitar sua capacidade de contra-argumentar colegas e, conseqüentemente, aumentar a sua capacidade de resistir a pressões grupais. Essas técnicas são baseadas em princípios da psicologia social e tem sido bastante efetivas na redução de comportamentos de risco, nesta faixa etária (Flay ${ }^{18}, 1985$; Bandura $^{3}$, 1986; Aronson ${ }^{2}$, 1988).

Dada a importância das variáveis familiares, no uso de anticoncepcionais, na adolescência, um outro ponto fundamental é o desenvolvimento de cursos de orientação sexual para pais de adolescentes que ficam sem saber como lidar com a sexualidade emergente de seus filhos, cada vez mais cedo. Há necessidade de conscientizar os pais de que a educação sexual não incita o comportamento sexual na adolescência (Steinberg ${ }^{40}, 1989$ ) e, dessa forma, diminuir a objeção dos mesmos contra a implementação de uma orientação sexual adequada, nesta etapa do desenvolvimento. Estudos apontam para o fato de que a comunicação entre mãe e filha pode ser um forte fator contribuinte para que a primeira relação sexual aconteça mais tarde (Strouse e Fabes ${ }^{41}$, 1985). Outras investigações ainda indicam que pais que têm um maior conhecimento sobre questões sexuais tendem a discutir mais esses assuntos com seus filhos quando comparados a pais que não possuem esse conhecimento (Fisher ${ }^{17}, 1986$ ).

Como aponta Furby e Beyth-Maron ${ }^{19}$ (1990), é fundamental que se melhore a estrutura social onde vive 0 adolescente, de maneira que ele possa fazer escolhas mais compativeis com uma vida adulta saudável. Esses mesmos autores também enfatizam que é importante haver conscientização de que, em nivel social, o momento é de ambivalência em relação à sexualidade. Adolescentes recebem constantemente duplas mensagens; a sociedade está de certa forma com dúvidas, ou seja, não sabe se deve desencorajar o adolescente da prática sexual ou, se maiores esforços devem ser colocados em prática quanto ao uso correto de anticoncepcionais, sobretudo do preservativo. Digman e col. ${ }^{15}$ (1985) sugerem que é necessário lembrar aos adolescentes que existem comportamentos sexuais intermediários, os quais podem levar à satisfação sem contudo se constituírem em comportamentos de risco para a gravidez e para a AIDS com a relação sexual desprotegida. Catania e col. ${ }^{9}$ (1990) também salientam que é essencial conscientizar as pessoas de que o uso de preservativos, bem como a realização de outras atividades de baixo risco, podem ser prazerosas e divertidas. 
Faz-se necessário que se desenvolva uma consciência mais crítica em relação ao caráter altamente autodestrutivo e ambivalente da sociedade em que vivemos. Ao mesmo tempo que se deve proteger o adolescente de comportamentos de auto-risco, é também de nossa responsabilidade não o tomar como "marginal man" (Lewin 25,1948$)$, acreditar que ele tem espaço e potencial para caminhar junto, na direção de um repensar crítico e reconstrutivo da sociedade em que ele vive.

BORUCHOVITCH, E. [Factors associated with the non-utilization of contraceptives in adolescence]. Rev. Saúde públ., S.Paulo, 26: 437-43, 1992. The literature concerning the major correlates of the non-utilization contraceptives in adolescence is critically reviewed. Research findings are analyzed and discussed in terms of their contribution not only to a deeper understanding of adolescents' risk-taking sexual behavior, but also to the implementation of more effective and realistic sexual education for this age-group.

Keywords: Adolescence. Contraceptive behavior. Sex education.

\section{Referênclas Bibllográficas}

1. ALVAREZ, M. de la Luz; BURROWS, R.; ZVAIGHAT, A.; MUZZO, S. Sociocultural characteristics of pregnant and nonpregnant adolescents of low socioeconomic status: a comparative study. Adolescence, 22 (85): 149-56, 1987.

2. ARONSON, E., The social animal. 5th ed. New York, W.H. Freeman and Co., 1988.

3. BANDURA, A. Self-efficacy mechanism in human agency. Amer. Psychol., 37: 122-47, 1986.

4. BAUMRIND, D. A developmental perspective on adolescent risk taking in contemporary America. New Dir. Child Dev. 37: 93-125, 1985.

5. BLACK, C. \& DEBLASSIE, R.R. Adolescent pregnancy: contributing factors, consequences, treatment, and plausible solutions. Adolescence, 20 (78): 281-90, 1985.

6. BROOKS-GUNN, J.; BOYER, C.B.; HEIN, K. Preventing HIV infection and AIDS in children and adolescents. Amer. Psychol., 43: 958-64, 1989.

7. BURGER, J.M. \& BURNS, L. The illusion of unique invulnerability and the use of effective contraception. Per. sonal. soc. Psychol. Bull., 14: 264-70, 1988.

8. BYRNE, D. Personality and attitudinal barriers to contraception. In: Byme, D. \& Fisher, W.A. eds. Adolescents, sex and contraception. Hilsdale Erlbaum, 1983. p. 3-31.

9. CATANIA, J.A.; KEGELES, S.M.; COATES, T.J. Towards an understanding of risk behavior: an AIDS reduction risk model (ARRM). Hith Educ. Quart., 17: 53-72, 1990.

10. CHILMAN, C.S. Adolescents sexuality in a changing American society. New York, John Wiley \& Sons, 1983.

11. DARABI, K.F.; JONES, J.; VARGA, P.; HOUSE, $M$. Evaluation of sex education outreach. Adolescence, 16 (63): 499-512, 1982

12. DAVIS, K. A theory of teenage pregnancy in the United States. In: Adolescent pregnancy and childbearing. Washington, D.C., Department of Health and Human Services, 1980
13. DEMBO, M.H., \& LUNDELL, B. Factors affecting adolescent contraception practices: implications for sex education. Adolescence, 16 (56): 657-64, 1979.

14. DICLEMENTE, R.J.; ZORN, J.; TEMOSHOK, L Adolescents and AIDS: a survey of knowledge, atritudes and beliefs about AIDS in San Francisco. Amer. J, publ. Hith, 76: 1143-5, 1986.

15. DIGNAN, M.; DENSON, D.; ANSPAUGH, D.; C'MCH, $D$. Effects of sex education on sexual behaviors of college students. Adolescence, 20 (77): 171-8, 1985.

16. ELKIND, D. Egocentrism in adolescence. Child Develop., 38, 1025-34, 1967.

17. FISHER, T.D. Parent-child communication about sex and young adolecents' sexual knwoledge and attitudes. Adolescence, 21 (83): 517-27, 1986.

18. FLAY, B.R. Psychosocial approaches to smoking prevention: a revew of findings. Hith Psychol, 4: 449-88, 1985.

19. FURBY, L. \& BEYTH-MAROM, R. Risk taking in adoles. cence: a decision-making perspective. Washington, D.C., The Camegie Council on Adolescent Development, 1990.

20. GOCHMAN, S. D. Some correlates of children's health beliefs and potential health behavior. J. Hlth soc. Behav., 12: $148-54,1971$.

21. GOCHMAN, S.D. Youngsters' health cognitions: crosssectional and longitudinal analyses. Hith behav. Syst. , 1986.

22. HART, B., \& HIITON, I. Dimensions of personality organization as predictors of teenage pregnancy risk. $J$. Pers. Assess., 52: 116-32, 1988.

23. INGERSOLL, G.M. \& ORR, D.P. Behavioral and emotional risk in early adolescents. J. early Adolesc., 9: 396 $408,1989$.

24. IRWIN Jr., C.E. \& MILLSTEIN, S.G. Biopsychosocial correlates of risk-taking behaviors during adolescence. $J$. Adolesc. Hith Care, 7: 825-965, 1986.

25. LEWIN, K. Resolving social conflicts. New York, Harper, 1948.

26. MASLACH, G., \& KERR, G. Tailoring sex-education programs to adolescents - a strategy for the primary prevention of unwanted adolescent pregnancies. Adolescence, 18 (70): 449-56, 1983.

27. MILLER, W.B. Sexual and contraceptive behavior in young unmarried women. Prim. Care., 3: 427-53, 1976.

28. MONSOUR, K. \& STEWART, B. Abortion and sexual behavior in college women. Amer.J. Orthopsychiat., 43: 804-14, 1973.

29. MONTEIRO, S.S.; REBELLO, S.M.; SCHALL, V.T. Zigzajds - an educational game about AIDS for children. Int. J. Hlth Educ., 10 (4): 32-35, 1991.

30. MORRISON, D.M. Adolescent contraceptive behavior: A review. Psychol. Bull., 98: 538-68, 1985.

31. NAMEROW, P.B.; LAWTON, A.I.; PHILLIBER, S.G. Teenagers' perceived and actual probabilities of pregnancy. Adolescence, 22 (86): 475-85, 1987.

32. PESTRAK, V.A., \& MARTIN, D. Cognitive development and aspects of adolescent sexuality. Adolescence, 20 (80): $981-7,1985$.

33. PHINNEY, V.G.; JENSEN, L.C.; OLSEN, J.A.; CUNDICK, B. Adolescence, 25 (98): 321-32, 1990.

34. PLAGET, J. Intellectual evolution from adolescence to adulthood. Humen Develop., 15: 1-12, 1972.

35. POLIT-O'HARA, D. \& KAHN, J. R. Communication and contraceptive practices in adolescent couples. Adolescence, $20(77): 33-42,1985$.

36. REBELLO, S. M.; MONTEIRO, S. S.; PINTO-DIAS, A.; MOREIRA, M. M.; SANTOS, M. G. \& SCHALL, V. T. O conhecimento da AIDS entre crianças e preadolescentes: um estudo amostral no Rio de Janeiro e em Belo Horizonte. Ciênc. e Cult., 41 (7): 151, 1989. 
37. ROMIG, C.A. \& BAKKEN, L. Teens at risk for pregnancy: the role of ego development and family processes. $J$. Adolesc., 13: 195-99, 1990.

38. ROSEN, R.H. \& AGER, J.W. Self-concept and contraception: preconception decision-making. Popul. Environ., 4: $11-23,1981$

39. SIMKINS, L. Consequences of teenage pregnancy and motherhood. Adolescence, 19 (73): 39-54, 1984

40. STENNBERG, L. Adolescence. 2nd ed. New York, McGraw-Hill, 1989.
41. STROUSE, J. \& FABES, R.A. Formal versus informal sources of sex education: competing forces in the sexual socialization of adolescents. Adolescence, 20: 251-3, 1985.

42. STRUNIN, L. \& HINGSON, R. Acquired immunodeficiency syndrome and adolescents: knowledge, beliefs and attitudes and behaviors. Pediatrics, 79: 825-8, 1987.

Recebido para publicaçđó em 20.5.1992

Reapresentado em 16.9.1992 Aprovado para publicação em 28,9.1992 